\title{
Optical Absorption of Water: Coulomb Effects versus Hydrogen Bonding
}

\author{
P. H. Hahn and W. G. Schmidt \\ Institut für Festkörpertheorie und -optik, Friedrich-Schiller-Universität, Max-Wien-Platz 1, 07743 Jena, Germany, \\ and Center for High Performance Simulation and Department of Physics, North Carolina State University, \\ Raleigh, North Carolina 27695-8202, USA \\ K. Seino, M. Preuss, and F. Bechstedt \\ Institut für Festkörpertheorie und -optik, Friedrich-Schiller-Universität, Max-Wien-Platz 1, 07743 Jena, Germany
}

J. Bernholc

Center for High Performance Simulation and Department of Physics, North Carolina State University, Raleigh, North Carolina 27695-8202, USA

(Received 3 October 2004; published 27 January 2005)

\begin{abstract}
The optical spectrum of water is not well understood. For example, the main absorption peak shifts upwards by $1.3 \mathrm{eV}$ upon condensation, which is contrary to the behavior expected from aggregationinduced broadening of molecular levels. We investigate theoretically the effects of electron-electron and electron-hole correlations, finding that condensation leads to delocalization of the exciton onto nearby hydrogen-bonded molecules. This reduces its binding energy and has a dramatic impact on the line shape. The calculated spectrum is in excellent agreement with experiment.
\end{abstract}

DOI: 10.1103/PhysRevLett.94.037404

The optical absorption of water is not well understood, despite decades of effort. The spectra of amorphous, hexagonal as well as cubic ice are dominated by a very pronounced first absorption peak at about $8.7 \mathrm{eV}[1,2]$, while the absorption spectrum of the liquid shows a similar structure [3,4]. In the case of cubic ice, the first absorption peak was attributed to the calculated density of states [5]. However, the universal occurrence of the $8.7 \mathrm{eV}$ peak in a variety of water phases suggests its molecular origin, as opposed to being due to specific transitions between electronic states arising from a crystalline structure with a particular symmetry. However, the lowest-lying excitation of the $\mathrm{H}_{2} \mathrm{O}$ molecule occurs at $7.4 \mathrm{eV}$ [6]. Intermolecular interactions are expected to induce a significant broadening of the energy levels relative to the isolated molecule. This should lead to a decrease of the transition energies relative to the molecular values [7-9]. Numerical studies of the optical properties of ice [10] based on the local density approximation, i.e., neglecting many-body effects beyond a mean-field description, indeed find the absorption onset at just below $6 \mathrm{eV}$.

The blueshift observed experimentally upon condensation of water molecules has been alternatively attributed to excitons of molecular origin [8,9] or to solvation and Rydbergization effects (destabilization of highly excited states in condensed phases by interactions with nearby molecules) [11]. However, an accurate description of optical spectra requires inclusion of many-body correlation effects from first principles, which is a difficult and computationally expensive task for complex disordered systems.

We exploit the recent progress in accurate modeling of optical properties using the many-body Green's function
PACS numbers: 78.20.Ci, 71.35.Cc, 78.40.Dw, 78.40.Pg

approach [12] to explain and quantitatively account for the peculiar absorption properties of solid water and $\mathrm{H}_{2} \mathrm{O}$ molecules. The results highlight the major role played by nearby hydrogen-bonded water molecules in affecting the spacial extent and the energetics of the exciton dominating the main absorption peak. It is clear that the exciton state "sees" nearby molecules due to its spacial extent and that its energetics will be affected by changes in the first and even the second coordination shell.

Naturally occurring hexagonal ice (ice-I $h$ ) is chosen as a model system. The spectra of cubic and amorphous ice, however, are very similar. We proceed in three steps: (i) We use density-functional theory in generalized gradient approximation (DFT-GGA) to obtain the structurally relaxed ground-state configuration of ice and the Kohn-Sham eigenvalues and eigenfunctions that enter the single- and two-particle Green's functions, (ii) the electronic quasiparticle spectrum is obtained within the GW approximation (GWA) [13] to the exchange-correlation self-energy, and (iii) the Bethe-Salpeter equation (BSE) is solved for coupled electron-hole excitations [14-16], thereby accounting for the screened electron-hole attraction and the unscreened electron-hole exchange [17-19].

The oxygen atoms in the ice-I $h$ structure lie on a hexagonal wurtzite lattice. Hydrogen atoms occupy the sites between neighboring oxygens in a disordered fashion but are subject to the ice rules; i.e., each oxygen is covalently bonded to two hydrogen atoms with the constraint that only one $\mathrm{H}$ atom can lie between two neighboring $\mathrm{O}$ atoms. We model the disorder within a periodically repeated supercell consisting of 16 molecules [20].

In detail, we start from first-principles pseudopotential calculations, using a massively parallel multigrid imple- 
mentation of the DFT-GGA [21]. The core electrons are eliminated with the use of pseudopotentials [22]. The spacing of the finest mesh used to describe the valence electron wave functions and charge density is $0.157 \AA$, and $16 \mathbf{k}$ points are used to sample the Brillouin zone. The mean-field effects of exchange and correlation in GGA are modeled using the Perdew, Burke, and Ernzerhof functional [23]. This functional is known to accurately reproduce the ice ground-state properties, including hydrogen bonding [24]. The Kohn-Sham energies calculated for the 16-molecule supercell are shown in Fig. 1. Because of intermolecular interactions, the occupied molecular states $2 a_{1}, 1 b_{2}, 3 a_{1}$, and $1 b_{1}$ broaden into energy bands. We calculate a band gap of $5.6 \mathrm{eV}, 0.6 \mathrm{eV}$ smaller than the lowest molecular transition energy. Earlier DFT studies found an energy gap of about $6 \mathrm{eV}$ for cubic ice [10] and $4.6 \mathrm{eV}$ for the liquid [7].

In the second step we include electronic self-energy effects. This requires the replacement of the GGA exchange and correlation potential by the nonlocal and energy-dependent self-energy operator $\Sigma\left(\mathbf{r}, \mathbf{r}^{\prime} ; E\right)$. We calculate $\Sigma$ in the GWA [13], where it is expressed as a convolution of the single-particle propagator $G$ and the dynamically screened Coulomb interaction $W$. As a further approximation, we use a model dielectric function [25] to calculate $W$. This speeds up the calculations substantially and results in quasiparticle energies that are within about $0.1-0.2 \mathrm{eV}$ of the complete calculations [26,27].

The electron-hole interaction is taken into account in the third step. The two-particle Hamiltonian,

$$
\begin{aligned}
H_{v c \mathbf{k}, v^{\prime} c^{\prime} \mathbf{k}^{\prime}}= & \left(\epsilon_{c \mathbf{k}}-\epsilon_{v \mathbf{k}}\right) \delta_{v v^{\prime}} \delta_{c c^{\prime}} \delta_{\mathbf{k}, \mathbf{k}^{\prime}}+2 \iint d \mathbf{r} d \mathbf{r}^{\prime} \psi_{c \mathbf{k}}^{*}(\mathbf{r}) \psi_{v \mathbf{k}}(\mathbf{r}) \bar{v}\left(\mathbf{r}-\mathbf{r}^{\prime}\right) \psi_{c^{\prime} \mathbf{k}^{\prime}}\left(\mathbf{r}^{\prime}\right) \psi_{v^{\prime} \mathbf{k}^{\prime}}^{*}\left(\mathbf{r}^{\prime}\right) \\
& -\iint d \mathbf{x} d \mathbf{r}^{\prime} \psi_{c \mathbf{k}}^{*}(\mathbf{r}) \psi_{c^{\prime} \mathbf{k}^{\prime}}(\mathbf{r}) W\left(\mathbf{r}, \mathbf{r}^{\prime}\right) \psi_{v \mathbf{k}}\left(\mathbf{r}^{\prime}\right) \psi_{v^{\prime} \mathbf{k}^{\prime}}^{*}\left(\mathbf{r}^{\prime}\right)
\end{aligned}
$$

describes the interaction of pairs of electrons in conduction states $|c \mathbf{k}\rangle$ and holes in valence states $|v \mathbf{k}\rangle$ [17-19]. The diagonal first part is given by the quasiparticle energies obtained in GWA. The second part, the electron-hole exchange term, where the short-range part of the bare Coulomb potential $\bar{v}$ enters, reflects the influence of local fields. Finally, the third part, which describes the screened electron-hole attraction, is calculated using the same approximations for $W$ as in the self-energy.

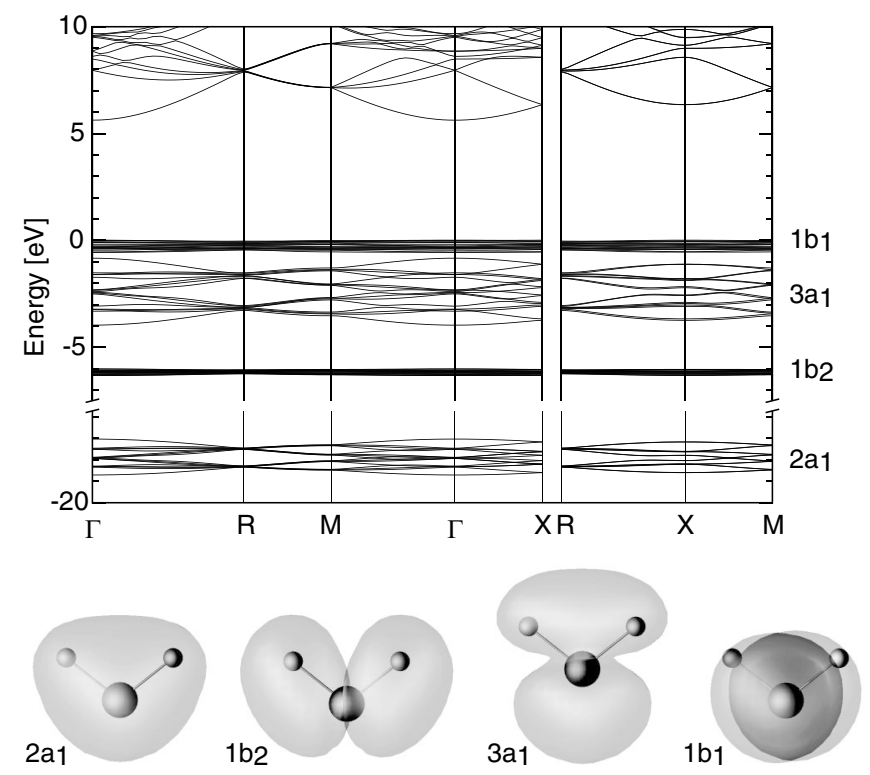

FIG. 1. Valence and conduction energy bands calculated in the DFT-GGA for the 16 molecule supercell. The occupied molecular states, from which the valence bands are derived, are shown below.
The eigenvalues and eigenvectors of the two-particle Hamiltonian (1) can be used to calculate the macroscopic dielectric function and thus the absorption spectrum. In our case, the Hamiltonian has been set up from 64 valence and 64 conduction bands using 40 random $\mathbf{k}$ points. In order to bypass the diagonalization of the Hamiltonian, we follow Glutsch et al. [28,29]: If the energy dependence of the macroscopic polarizability on the eigenvalues of the exciton Hamiltonian is Fourier transformed, the polarizability can be obtained from the solution of an initial-value problem for the vector $|\mu(t)\rangle$. Its time evolution is driven by the pair Hamiltonian (1)

$$
i \hbar|\dot{\mu}(t)\rangle=H|\mu(t)\rangle .
$$

The initial values of the vector elements are given by

$$
\mu_{c v \mathbf{k}}^{i}(0)=\frac{\left\langle c \mathbf{k}\left|v_{i}\right| v \mathbf{k}\right\rangle}{\epsilon_{c \mathbf{k}}^{\mathrm{GGA}}-\epsilon_{v \mathbf{k}}^{\mathrm{GGA}}}
$$

where $v_{i}$ is the $i(=x, y, z)$ component of the velocity operator and the energy differences in the denominator are calculated within GGA. The macroscopic dielectric function with the broadening parameter $\gamma$ is then obtained by the Fourier transform of $e^{-\gamma t} \cdot\langle\mu(0) \mid \mu(t)\rangle$.

In Fig. 2 we compare the optical spectra calculated according to these three levels of theory with experiment [2]. The spectrum obtained within the DFT-GGA agrees with earlier independent-particle results [10]. The calculated onset of absorption occurs at too low energies (below $6 \mathrm{eV}$ ), and the first absorption maximum at about $8 \mathrm{eV}$ is far less pronounced than the corresponding feature in experiment. The inclusion of the many-body electronelectron interaction in the GWA, i.e., the electronic selfenergy, leads to a nearly rigid blueshift of the spectrum, 
severely overestimating the energy positions of the measured peaks. The Coulomb correlation of electrons and holes, accounted for by solving the BSE, leads to the appearance of a sharp excitonic peak below the onset of the vertical quasiparticle transition energies. The peak positions and the line shape obtained from the BSE agree well with experiment. Since no input parameters to the calculation have been taken from experiment or fitted, the agreement between theory and measurement is truly impressive.

After having reproduced the main features of the optical absorption of ice, we explore the origin of the prominent first peak. To this end we calculate the optical transitions of gas-phase $\mathrm{H}_{2} \mathrm{O}$ molecules. The GWA/BSE calculations are performed as for the solid, but the frequency-dependent inverse dielectric matrix is now computed in the independent-particle approximation. At the single-particle level of theory, i.e., in the DFT-GGA, an energy of $6.2 \mathrm{eV}$ is obtained for the lowest singlet pair excitation. The electronic self-energy blueshifts this value by $6.3 \mathrm{eV}$ to $12.5 \mathrm{eV}$. This is partially compensated by an exciton binding energy of $5.3 \mathrm{eV}$, leading finally to an optical absorption at $7.2 \mathrm{eV}$, in excellent agreement with the experimental value of $7.4 \mathrm{eV}$ [6]. These results can be compared with those for ice, shown in Fig. 2, where we find for the first absorption peak a self-energy shift of about $4.5 \mathrm{eV}$ and an exciton binding energy (related to the position of the first major peak of the vertical quasiparticle transitions) of $3.2 \mathrm{eV}$. Self-energy and excitonic effects in ice are thus reduced by $29 \%$ and $40 \%$, respectively, compared to gas-phase molecules. The reduction of the self-energy and the exciton binding energy upon condensation of the molecules is

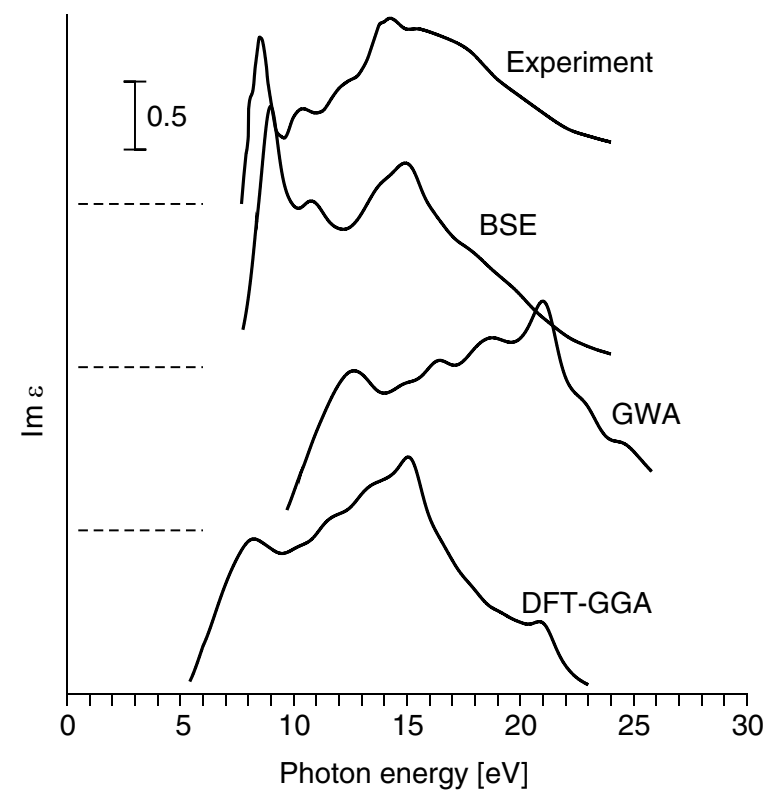

FIG. 2. The imaginary part of the dielectric function of hexagonal ice measured at $80 \mathrm{~K}$ (from Ref. [2]) and calculated within the DFT-GGA, the GWA, and from the BSE. expected, due to the larger screening in the solid. The fact, however, that the exciton binding energy is more affected than the self-energy points to an additional effect, namely, a change in the localization of the exciton. The attractive interaction between an electron and a hole is inversely proportional to their average distance, the socalled exciton radius

$$
R=\int d \mathbf{r}_{e} \int d \mathbf{r}_{h}\left|\mathbf{r}_{e}-\mathbf{r}_{h}\right|\left|\Psi\left(\mathbf{r}_{e}, \mathbf{r}_{h}\right)\right|^{2},
$$

where $\Psi\left(\mathbf{r}_{e}, \mathbf{r}_{h}\right)$ is the electron-hole pair wave function depending on the positions $\mathbf{r}_{e}$ and $\mathbf{r}_{h}$ of electrons and holes, respectively. The spatial distribution of electron-hole pairs therefore influences their energy.

In the case of an isolated molecule the localization of the electron-hole pair is immediately obtained from the spatial extension of the respective molecular orbitals, which relax upon optical excitation. The highest occupied molecular orbital (HOMO) of water is a nonbonding oxygenlocalized $1 b_{1}$ orbital. It barely changes upon excitation of one electron into the lowest unoccupied molecular orbital (LUMO). The latter, however, is strongly affected by partial occupation. The LUMO shows a significant probability density in the proximity of the $\mathrm{O}$ atom, as well as two lobes that protrude from the molecule in the proton directions; see Fig. 3(a). Optical excitation of one electron from the HOMO into the LUMO leads to a considerable expansion of these lobes, as shown in Fig. 3(b). Nevertheless, due to the attractive interaction with the hole at the oxygen atom, the electron remains close to the molecule. We calculate an average electron-hole distance $R$ of $2.27 \AA$.

How does the spatial distribution of the excited electrons change upon condensation of the $\mathrm{H}_{2} \mathrm{O}$ molecules? The solution of the BSE yields correlated electron-hole pair states. The pair wave functions $\Psi\left(\mathbf{r}_{e}, \mathbf{r}_{h}\right)$ are scalar func-

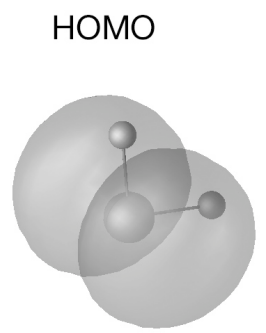

(a)
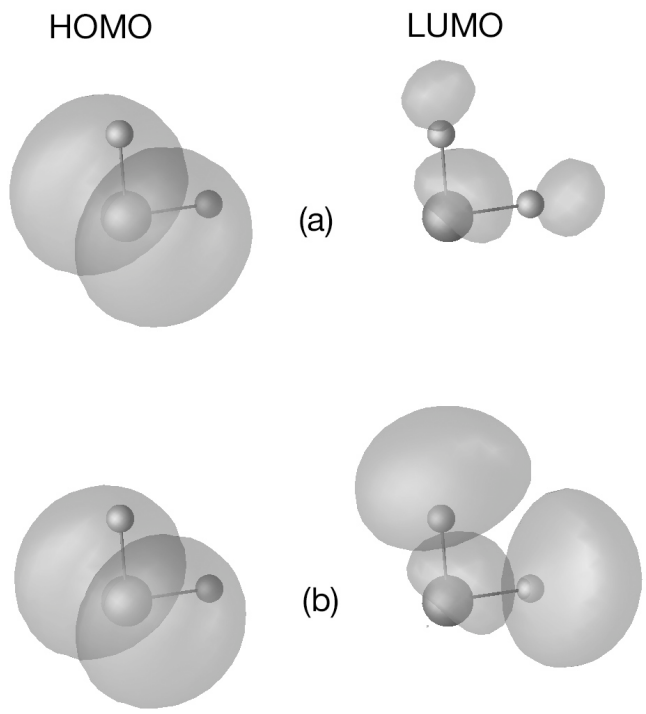

FIG. 3. HOMO and LUMO of the gas-phase water molecule in the ground state (a) and upon optical excitation (b). 


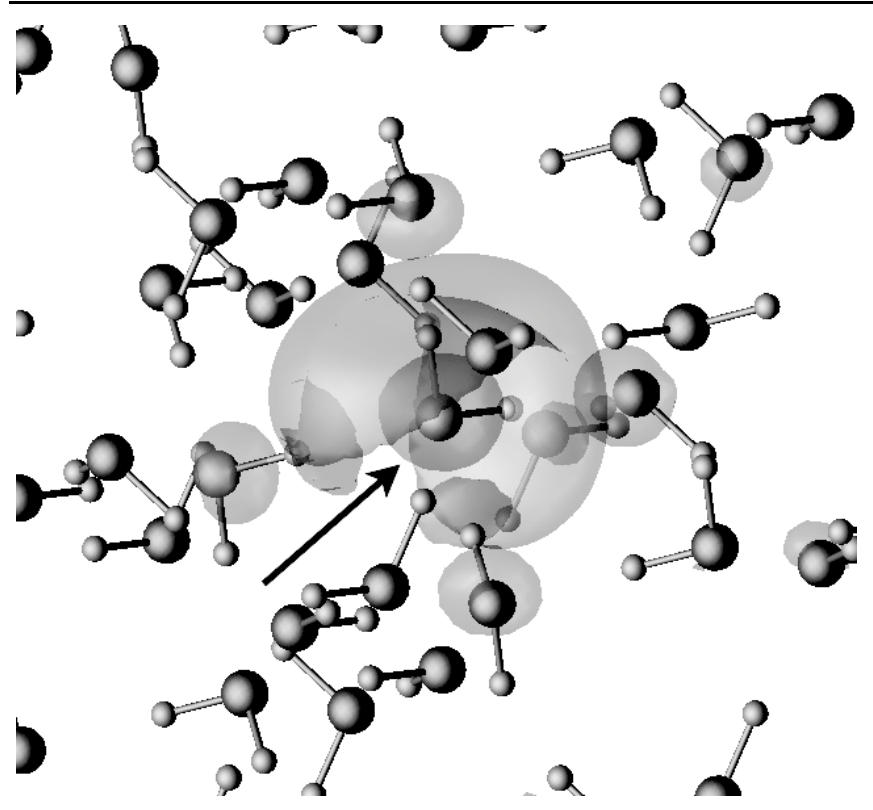

FIG. 4. Spatial distribution of the electron in the exciton associated with the lowest optical absorption peak of ice-I $h$. The hole is located at the oxygen position marked with an arrow (see text).

tions in the two-particle space. The center of gravity of the hole belonging to the exciton wave function responsible for the first absorption peak is close to an oxygen position $\tau_{\text {oxygen }}$. In Fig. 4 we show the probability density $\left|\Psi\left(\mathbf{r}_{e}, \mathbf{r}_{h}=\tau_{\text {oxygen }}\right)\right|^{2}$ corresponding to the electron distribution of that particular exciton. It is still reminiscent of the molecular LUMO shown in Fig. 3, but it expanded considerably farther towards the nearest-neighbor molecules. The calculated mean distance $R$ between the electron and the hole is $4.02 \AA$, much larger than in the case of a gasphase water molecule.

This finding provides an intuitive explanation for the surprising energy shift discovered in experiments: The matrix of surrounding $\mathrm{H}_{2} \mathrm{O}$ molecules enables the optically excited electron to move farther away from the hole left behind at the oxygen atom, thus reducing the binding energy of the electron-hole pair from 5.3 to $3.2 \mathrm{eV}$. This effect overcompensates the narrowing of the HOMOLUMO energy gap resulting from the condensation of the $\mathrm{H}_{2} \mathrm{O}$ molecules and leads to the observed blueshift of the optical absorption. The sensitivity of the molecular exciton to hydrogen-bonded neighbor molecules suggests the possibility of detecting changes in the solvation shell due to foreign molecules or ions by means of optical spectroscopy. More generally, the calculations show that the optically excited states of water molecules respond far more sensitively to intermolecular interactions than the ground state of their electronic structure.

Generous grants of computer time from the LeibnizRechenzentrum München, the HöchstleistungsRechenzentrum Stuttgart, and the DoD Challenge
Program are gratefully acknowledged. We thank the Deutsche Forschungsgemeinschaft (SCHM-1361/6), the NSF, and the DOE for financial support.

[1] R. Onaka and T. Takahashi, J. Phys. Soc. Jpn. 24, 548 (1968).

[2] K. Kobayashi, J. Phys. Chem. 87, 4317 (1983).

[3] G. D. Kerr, R. N. Hamm, M. W. Williams, R. D. Birkhoff, and L. R. Painter, Phys. Rev. A 5, 2523 (1972).

[4] H. Hayashi, N. Watanabe, Y. Udagawa, and C.-C. Kao, Proc. Natl. Acad. Sci. U.S.A. 97, 6264 (2000).

[5] V. F. Petrenko and I. A. Ryzhkin, Phys. Rev. Lett. 71, 2626 (1993).

[6] W. F. Chan, G. Cooper, and C. E. Brion, Chem. Phys. 178, 387 (1993).

[7] K. Laasonen, M. Sprik, M. Parrinello, and R. Car, J. Chem. Phys. 99, 9080 (1993).

[8] G. P. Parravicini and L. Resca, Phys. Rev. B 8, 3009 (1973).

[9] L. Resca and R. Resta, Phys. Status Solidi (b) 81, 129 (1977).

[10] W. Y. Ching, M.-Z. Huang, and Y.-N. Xu, Phys. Rev. Lett. 71, 2840 (1993).

[11] B. D. Bursulaya, J. Jeon, C.-N. Yang, and H. J. Kim, J. Phys. Chem. A 104, 45 (2000).

[12] G. Onida, L. Reining, and A. Rubio, Rev. Mod. Phys. 74, 601 (2002).

[13] M. S. Hybertsen and S. G. Louie, Phys. Rev. B 34, 5390 (1986).

[14] S. Albrecht, L. Reining, R. Del Sole, and G. Onida, Phys. Rev. Lett. 80, 4510 (1998).

[15] L. X. Benedict, E. L. Shirley, and R. B. Bohn, Phys. Rev. Lett. 80, 4514 (1998).

[16] M. Rohlfing and S. G. Louie, Phys. Rev. Lett. 83, 856 (1999).

[17] L. J. Sham and T. M. Rice, Phys. Rev. 144, 708 (1966).

[18] W. Hanke and L. J. Sham, Phys. Rev. B 12, 4501 (1975).

[19] W. Hanke and L. J. Sham, Phys. Rev. B 21, 4656 (1980).

[20] I. Morrison, J.-C. Li, S. Jenkins, S. S. Xantheas, and M. C. Payne, J. Phys. Chem. B 101, 6146 (1997).

[21] E. L. Briggs, D. J. Sullivan, and J. Bernholc, Phys. Rev. B 54, 14362 (1996).

[22] N. Troullier and J.L. Martins, Phys. Rev. B 43, 1993 (1991).

[23] J. P. Perdew, K. Burke, and M. Ernzerhof, Phys. Rev. Lett. 77, 3865 (1996).

[24] D. R. Hamann, Phys. Rev. B 55, R10157 (1997).

[25] F. Bechstedt, R. Del Sole, G. Cappellini, and L. Reining, Solid State Commun. 84, 765 (1992).

[26] J.E. Northrup, Phys. Rev. B 47, R10032 (1993).

[27] W. G. Schmidt, S. Glutsch, P. H. Hahn, and F. Bechstedt, Phys. Rev. B 67, 085307 (2003).

[28] S. Glutsch, D. S. Chemla, and F. Bechstedt, Phys. Rev. B 54, 11592 (1996).

[29] P. H. Hahn, W. G. Schmidt, and F. Bechstedt, Phys. Rev. Lett. 88, 016402 (2002). 